\title{
INVERSE FEL PROTON ACCELERATOR VIA PERIODICALLY MODULATED CRYSTAL STRUCTURE
}

\author{
S. A. BOGACZ \\ Accelerator Physics Department, Fermi National Accelerator Laboratory* \\ P. O. Box 500, Batavia, IL 60510
}

(Received 27 May 1993; in final form 28 June 1993)

\begin{abstract}
Presented study explores the idea of using a visible light wave to accelerate relativistic protons via the inverse FEL mechanism. Here, a strain modulated crystal structure - the superlattice - plays the role of a microscopic undulator providing very strong ponderomotive coupling between the beam and the light wave. Purely classical treatment of relativistic protons channeling through a superlattice is performed in a self-consistent fashion involving the Maxwell wave equation for the accelerating electromagnetic field and the relativistic Boltzmann equation for the protons. It yields the accelerating efficiency in terms of the negative gain coefficient for the amplitude of the electromagnetic wave - the rate the energy is extracted from the light by the beam. The analytic formalism presented allows one to find the acceleration rate in a simple closed form, which is further evaluated for a model beam-optical cavity system to verify the feasibility of our scheme.
\end{abstract}

KEY WORDS: Electromagnetic Field Calculations, Free-Electron Lasers, Laser-Beam Accelerators, Radiation

\section{INTRODUCTION}

Here we suggest using a solid-state superlattice as an undulator in conjunction with an optical pumping cavity to accelerate relativistic protons. Heavier particles are more appropriate than electrons or positrons, because they are not susceptible to emitting photons via spontaneous synchrotron radiation and therefore are more likely to absorb energy from an electromagnetic wave. The idea of the inverse FEL mechanism employed to accelerate charged particles ${ }^{1}$ is not new by itself; what we propose here is to replace a conventional magnetic undulator with a microscopically modulated crystal structure - the superlattice, which assures much stronger ponderomotive coupling to the pumping wave. Furthermore, its microscopic undulator periodicity shifts the wavelength of the pumping optical mode to much shorter waves - it opens a possibility of accelerating the beam with high power laser in the visible region.

Such periodic crystal structures occur naturally in several alloy systems or may be prepared artificially with vapor deposition techniques ${ }^{2}$. We consider a superlattice

\footnotetext{
* Operated by the Universities Research Association, Inc., under a contract with the U.S. Department of Energy
} 


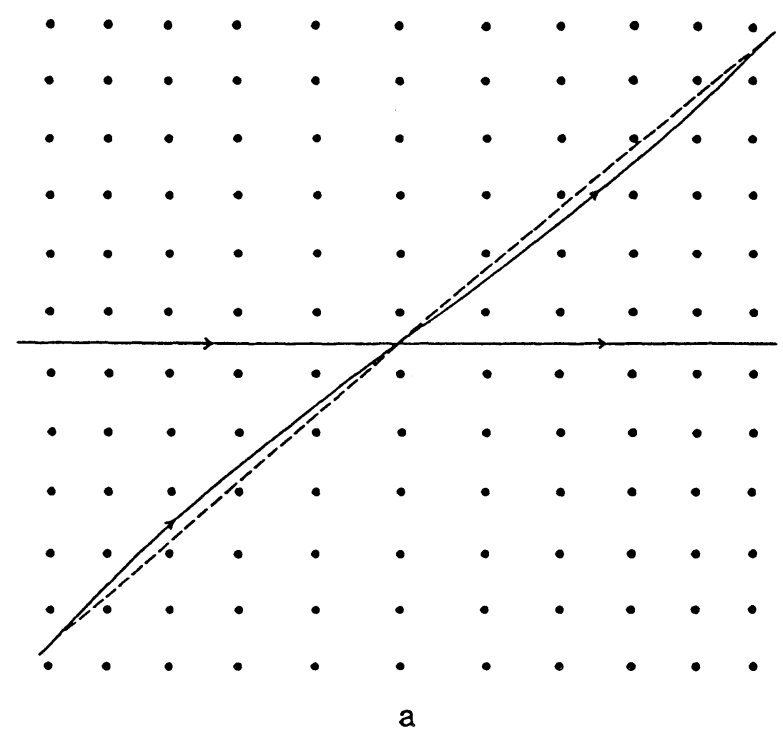

FIGURE 1(a):

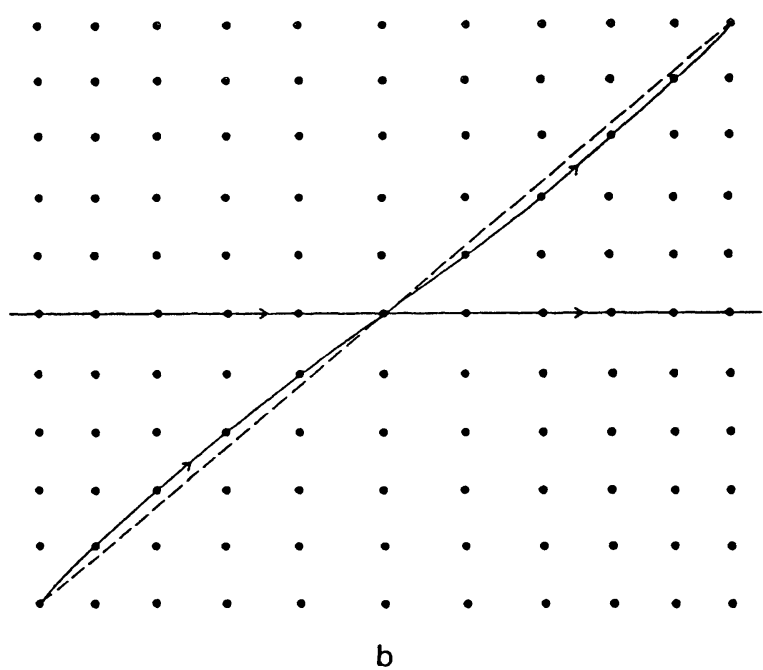

FIGURE 1(b):

FIGURE 1 Center of the channeling trajectory for a [110] direction in a strain-modulated superlattice; a) for protons (located in a low density region),

b) for $\mathrm{H}^{-}$(located in a high density region).

The [100] channeling direction yields no undulator effect. 
with an accompanying strain modulation, which is a natural consequence of the two constituents having slightly different lattice spacings (eg. Si-Ge superlattice).

The main idea of using a modulated crystal structure as an undulator is illustrated schematically in Figure 1. A beam of relativistic particles, while channeling through the crystal, follows a well-defined trajectory. This treatment will be limited to protons but could be modified for negative ions (eg. $\mathrm{H}^{-}$), as well provided that the stripping probability for $\mathrm{H}^{-}$is not too high. Figure 1 depicts channeling paths, which for protons would lie in low density regions of the crystal and conversely for $\mathrm{H}^{-}$. There are two distinct channeling directions: parallel to the superlattice growth direction and also at a $45^{\circ}$ angle to this direction. The latter interests us here because, as may be seen from Figure 1, the center of the channeling axis is modulated by the superlattice periodicity. This is the essence of the solid state undulator: the particles are periodically accelerated perpendicular to their flight path as they traverse the channel. The undulator wavelengths typically fall in the range $50-500 \AA$, far shorter than those of any macroscopic undulator. Furthermore, the electrostatic crystal fields involve the line-averaged nuclear field and can be two or more orders of magnitude larger than the equivalent fields of macroscopic magnetic undulators (translated into the corresponding electric field in the rest frame of a relativistic particle). Both of these factors hold the promise of greatly enhanced coupling between the beam and the pumping electromagnetic wave.

\section{CHANNELING OF RELATIVISTIC PROTONS THROUGH A STRAIN- MODULATED SUPERLATTICE}

Following the spirit of the Vlasov equation ${ }^{3}$ we will describe a high-intensity proton beam in terms of a classical distribution function, $f(\mathbf{p}, \mathbf{r}, t)$, governed by the relativistic Boltzmann equation. The transverse dynamics of relativistic protons propagating in a strain-modulated superlattice is modeled by a harmonic crystal field potential ${ }^{4}$ and leads to generation of a transverse current. This couples the Vlasov equation to the Maxwell wave equation. Therefore, the problem reduces to a self-consistent solution of the Vlasov and the wave equations, which will be treated in detail in the next few sections. Finally, a closed analytic expression for the amplitude gain/loss coefficient will be obtained in the linear approximation for the appropriate regime with respect to the cavity length and the momentum spread in the incident beam.

We start with a relativistic Lagrangian describing motion of a proton in an arbitrary electromagnetic field $(\mathbf{A}, \phi)$ :

$$
L=-m c^{2} \sqrt{1-v^{2} / c^{2}}+\frac{e}{c} \mathbf{v} \cdot \mathbf{A}-e \phi .
$$

Here $\mathbf{A}$ is a vector potential of an electromagnetic field and $\phi$ is a phenomenological harmonic crystal-field potential, which describes both transverse focusing of the beam and longitudinal modulation of the minimum of the harmonic potential well. More realistic description of the planar channeling is usually given in terms of the Moliere potential $^{5}$, which accounts for the averaged electric field of the ions and electron 
cloud around each lattice site. For the purpose of our model calculation, a harmonic approximation to the focusing potential is quite sufficient, and it can be written as follows:

$$
\phi=\phi_{0}+\frac{1}{2} \phi_{1}\left(x-x_{1} \cos g z\right)^{2},
$$

where $g=2 \pi / \ell$, is the strain modulation periodicity and $x_{1}, \phi_{1}, \phi_{0}$ are parameters of the potential. In order to avoid unnecessary complexity we choose to work directly in the laboratory reference frame and use a space-time description of the problem.

We introduce the canonical momentum of a particle

$$
p^{\alpha}=\frac{\partial L}{\partial \dot{x}^{\alpha}}=m \gamma v^{\alpha}+\frac{e}{c} A^{\alpha}
$$

and write the Hamiltonian as

$$
H=p^{\alpha} \dot{x}^{\alpha}-L=m \gamma c^{2}+e \phi,
$$

with

$$
\gamma(\mathbf{p})=\sqrt{1+\frac{1}{(m c)^{2}}\left(\mathbf{p}-\frac{e}{c} \mathbf{A}\right)^{2}} .
$$

Hamilton's equations of motion are then

$$
\begin{gathered}
\dot{x}^{\alpha}=\frac{\partial H}{\partial p^{\alpha}}=\frac{1}{m \gamma}\left(p^{\alpha}-\frac{e}{c} A^{\alpha}\right) \\
\dot{p}^{\alpha}=-\frac{\partial H}{\partial x^{\alpha}}=\frac{e}{m c \gamma}\left(p^{\beta}-\frac{e}{c} A^{\beta}\right) \frac{\partial A^{\beta}}{\partial x^{\alpha}}-e \frac{\partial \phi}{\partial x^{\alpha}} .
\end{gathered}
$$

A beam of protons moving along the $z$ axis can be described in terms of a distribution function, $f(\mathbf{p}, \mathbf{x}, t)$; this distribution function obeys the relativistic Vlasov equation

$$
\frac{\partial f}{\partial t}+\frac{1}{m \gamma}\left(p^{\alpha}-\frac{e}{c} A^{\alpha}\right) \frac{\partial f}{\partial x^{\alpha}}+\left[\frac{e}{m c \gamma}\left(p^{\beta}-\frac{e}{c} A^{\beta}\right) \frac{\partial A^{\beta}}{\partial x^{\alpha}}-e \frac{\partial \phi}{\partial x^{\alpha}}\right] \frac{\partial f}{\partial p^{\alpha}}=I\left(p^{\alpha}\right) .
$$

Here $\mathrm{I}\left(p^{\alpha}\right)$ is a collision integral accounting for various incoherent proton scattering processes. This equation will be treated iteratively and only linear terms in the A-field will be retained. In the zero-order solution, $\mathbf{A}=\mathbf{0}$ and the corresponding distribution function $f=f^{(0)}$ is obtained (in the absence of collisions) from the solution of

$$
\frac{\partial f^{(0)}}{\partial t}+\frac{p^{\alpha}}{m \gamma} \frac{\partial f^{(0)}}{\partial x^{\alpha}}-e \frac{\partial \phi}{\partial x^{\alpha}} \frac{\partial f^{(0)}}{\partial p^{\alpha}}=0
$$


A class of solutions, $f^{(0)}$, describing a beam of protons with a sharply peaked initial momentum distribution, $\Lambda\left(p_{z}-p_{0}\right), p_{x}=0$ can be easily constructed by solving the following set of equations:

$$
\begin{gathered}
\dot{p}_{x}=-e \phi_{1}\left(x-x_{1} \cos g z\right), \\
\dot{p}_{z}=-e \phi_{1}\left(x-x_{1} \cos g z\right) x_{1} g \sin g z .
\end{gathered}
$$

Keeping only linear terms in $x_{1}$, yields the general solution (including both transient and steady-state parts) as follows:

$$
x=\frac{x_{1}}{1-U^{2}} \cos (g z+\theta)+C \cos k_{\beta} z
$$

and

$$
\dot{p}_{z}=0,
$$

where $U=g / k_{\beta}$ and $C$ is an arbitrary constant defining the initial amplitude of the transient oscillation. Here, $k_{\beta}$ is a focussing strength of the crystal channel given explicitly by

$$
k_{\beta}=\sqrt{\frac{e \phi_{1}}{p_{z} v_{z}}} .
$$

The remaining phase shift, $\theta$, depends on the relative strength of the two frequencies $k_{\beta}$ and $g$. Its asymptotic behavior can be summarized as follows

$$
\begin{array}{lll}
\theta \rightarrow 0 & \text { for } & g \ll k_{\beta} \\
\theta \rightarrow \pi & \text { for } & k_{\beta} \ll g .
\end{array}
$$

It follows from Equations (2.12)-(2.14) that the initial distribution of canonical momenta in the $z$ direction is conserved during the channeling, while the particles follow the trajectory defined by $x^{(0)}(z)=x$. In principle the trajectory is out of phase with the driving strain modulation (in the extreme relativistic regime $\theta$ reaches $\pi$ ). This implies that particles entering the crystal with $x$ coordinates that are off the channel plane lose energy via scattering mechanisms and asymptotically approach the channel plane. ${ }^{6}$ Therefore, the solution, $f^{(0)}$, can be factorized as follows:

$$
f^{(0)}=n_{0} \delta\left(x-x^{(0)}\right) \delta\left(p_{x}-p^{(0)}\right) \Lambda\left(p_{z}-p_{0}\right),
$$

where

$$
p_{x}^{(0)}=p_{z} \frac{\partial x^{(0)}}{\partial z}=-p_{z} x^{(0)} \frac{g x_{1}}{1-U^{2}} \sin g z .
$$


Here $\Lambda\left(p_{z}-p_{0}\right)$ describes an initial momentum distribution and $n_{0}$ is a concentration of particles per unit area of the channeling plane. The distribution function, $f^{(1)}$, generated by the A-field obeys the following linearized Vlasov equation:

$$
\begin{aligned}
\frac{\partial f^{(1)}}{\partial t} & +\frac{p_{x}}{m \gamma} \frac{\partial f^{(1)}}{p d x}-\frac{1}{m \gamma} \frac{e}{c} A_{x} \frac{\partial f^{(0)}}{\partial x}+\frac{p_{z}}{m \gamma} \frac{\partial f^{(1)}}{\partial z} \\
& +\frac{e p_{x}}{m c \gamma} \frac{\partial A_{x}}{\partial z} \frac{\partial f^{(1)}}{\partial p_{x}}-e \frac{\partial \phi}{\partial x} \frac{\partial f^{(1)}}{\partial p_{x}}=\frac{f^{(1)}}{\tau} .
\end{aligned}
$$

Here we have used a relaxation time approximation for the collision integral, modelling it by the relaxation time $\tau$. We have also assumed that only the transverse component of the A-field is present and $A_{x} \equiv A(z, t)$. We seek a solution, $f^{(1)}$, in the following form:

$$
f^{(1)}=n_{0} \delta\left(x-x^{(0)}\right) \delta\left(p_{x}-p_{x}^{(0)}\right) h\left(z, p_{z}, t\right),
$$

where $h$ describes bunching of particles due to the presence of the A-field. Substituting Equations (2.15)-(2.16) and (2.18) into Equation (2.17) leads to the following kinetic equation for $h$ :

$$
\frac{\partial h}{\partial t}+\frac{p_{z}}{m \gamma} \frac{\partial h}{\partial z}+\frac{e}{c} \frac{p_{z}}{m \gamma} \frac{g x_{1}}{1-U^{2}} \frac{\partial A}{\partial z} \frac{\partial \Lambda}{\partial p_{z}} \sin g z=-\frac{h}{\tau} .
$$

The inhomogeneous term in Equation (2.19) plays the role of a driving force representing acceleration of the particles by the ponderomotive force due to the transverse motion (induced by the crystal field) in the presence of the A-field. The transverse current induced by the fields is given by

$$
j_{x}=e \int_{-\infty}^{\infty} d p_{x} \int_{-\infty}^{\infty} d p_{z} v_{x} f^{(1)}
$$

where

$$
v_{x}=\frac{1}{m \gamma}\left(p_{x}-\frac{e}{c} A\right) .
$$

The current in a single channel can be written to linear accuracy in A as follows:

$$
j_{x}=-n_{0} e \delta\left(x-x^{(0)}\right) \int_{-\infty}^{\infty} d p_{z} h\left(z, p_{z}, t\right) \frac{p_{z}}{m \gamma} \frac{g x_{1}}{1-U^{2}} \sin g z .
$$

The net transverse current in the crystal is given by a sum of discrete planar currents concentrated at each channeling site of the $x$ direction. According to the Appendix, an infinite array of equally spaced currents is equivalent to a smoothed uniform current distribution given by Equation (2.22) but without the factor $\delta\left(x-x^{(0)}\right)$ and with an 
associated proton density redefined as $n=n_{0} / a$, where $a$ is the spacing between adjacent channels. A more general situation where the crystal field potential in each channel is shifted in phase is also discussed in the Appendix. The resulting transverse current couples Equation (2.19) to the following wave equation:

$$
\left(\frac{\partial^{2}}{\partial z^{2}}-\frac{1}{c^{2}} \frac{\partial^{2}}{\partial t^{2}}\right) A=\frac{4 \pi n e}{c} \int_{-\infty}^{\infty} d p_{z} h \frac{p_{z}}{m \gamma} \frac{g x_{1}}{1-U^{2}} \sin g z
$$

resulting in a closed system of equations for $h$ and $A$. Here the A-field can be identified as a sum of the macroscopic driving field and a self-consistent electromagnetic field propagating in the crystal structure. We will not attempt to solve this system of partial-differential-integral equations exactly. For the purpose of calculating a linear amplitude loss/gain coefficient, it is sufficient to confine the solution for the A-field to only the first step of the iteration procedure. The procedure is analogous to the Born approximation in scattering theory. ${ }^{7}$ We start with a single plane-wave solution of arbitrary $\omega$ and $k$ propagating in free space along the $z$ axis in both directions and use it as a zero order iteration step:

$$
A_{ \pm}^{(0)}=A_{0} e^{-i \omega t \pm i k z}
$$

Putting $A=A_{ \pm}^{(0)}$ in Equation (2.19), one can solve it analytically for $h=h_{ \pm}^{(1)}$ by constructing a Green's function with the appropriate boundary conditions built in. We consider a finite crystal undulator extending along the $z$ axis from 0 to $L$. The bunching function, $h_{+}^{(1)}$, corresponding to the right-propagating initial wave, $A_{+}^{(0)}$, vanishes outside the undulator and it is assumed to be continuous at the entry point, $z=0$, of the $A_{+}^{(0)}$ wave. Similarly for the leftgoing solution, $A_{-}^{(0)}$, the analogous initial condition is satisfied at its entry point, $z=L$. The complete set of boundary conditions can be written in the following compact notation:

$$
h_{ \pm}^{(1)}\left(z_{ \pm}\right)=0
$$

where

$$
z_{+}=0, \quad z_{-}=L
$$

Finally, the solution of Equation (2.19) with the boundary condition from Equations (2.25)-(2.26) is expressed in the following integral form:

$$
h_{ \pm}^{(1)}(z)=\int_{z_{ \pm}}^{z} d z^{\prime} H_{ \pm}^{(1)}\left(z^{\prime}\right) e^{i\left(z-z^{\prime}\right) m \gamma \omega / p_{z}}
$$

where

$$
H_{ \pm}^{(1)}(z)= \pm i k A_{ \pm}^{(0)}(z) \sin g z Q \frac{\partial \Lambda}{\partial p_{z}}
$$


and

$$
Q=\frac{e}{c} \frac{g x_{1}}{1-U^{2}} .
$$

Collisions are modeled by writing $\omega \rightarrow \omega+i / \tau$. Substituting the above solution for $h_{ \pm}^{(1)}$ in Equation (2.23) reduces it to an inhomogeneous Helmholtz equation for $A_{ \pm}^{(0)}$ :

$$
\left(\frac{\partial^{2}}{\partial z^{2}}-\frac{1}{c^{2}} \frac{\partial^{2}}{\partial t^{2}}\right) A_{ \pm}^{(1)}(z)=J_{ \pm}(z)
$$

where the explicit formula for $J_{ \pm}$can be obtained from Equations (2.27)-(2.29). To solve Equation (2.30) one may integrate it with the following boundary conditions at the entry points for the right- and left- propagating solutions

$$
A_{ \pm}^{(0)}\left(z_{ \pm}\right)=A_{ \pm}^{(1)}\left(z_{ \pm}\right)
$$

and

$$
\left.\frac{\partial}{\partial z} A_{ \pm}^{(0)}\right|_{z=z_{ \pm}}=\left.\frac{\partial}{\partial z} A_{ \pm}^{(1)}\right|_{z=z_{ \pm}}
$$

The solution can be written explicitly in terms of the Green's function for the Helmholtz equation

$$
A_{ \pm}^{(1)}(z)=A_{ \pm}^{(0)}(z)+\int_{z_{ \pm}}^{z_{\mp}} d z^{\prime} \frac{e^{i k\left|z-z^{\prime}\right|}}{2 k i} J_{ \pm}\left(z^{\prime}\right), k=\omega / c
$$

where

$$
J_{ \pm}(z)= \pm i k \int_{-\infty}^{\infty} d p_{z} F\left(p_{z}\right) \sin g z \int_{z_{ \pm}}^{z} d z^{\prime} \sin g z^{\prime} A_{ \pm}^{(0)}\left(z^{\prime}\right) e^{i\left(z-z^{\prime}\right) m \gamma \omega / p_{z}}
$$

with the kernel $F\left(p_{z}\right)$ defined as follows

$$
F\left(p_{z}\right)=4 \pi n Q^{2} \frac{p_{z}}{m \gamma} \frac{\partial \Lambda}{\partial p_{z}} .
$$

Carrying out the integration in Equation (2.34) leads to the required solutions describing the evolution of the right- and left- propagating waves. To introduce a singlepass loss/gain coefficient, one should examine the resulting amplitudes after passing through an undulator of length $L$, i.e., evaluate $A_{ \pm}^{(0)}\left(z=z_{\mp}\right)$ by using Equation (2.34). On the other hand, one can model the effect of coupling by adding a small complex part $i \kappa^{ \pm}=\alpha^{ \pm}+i \beta^{ \pm}$to the $k$-vector; here $\alpha$ is a gain/loss coefficient and $\beta$ describes a small shift in the phase velocity of the optical mode. This could be summarized by the following expression

$$
A_{ \pm}^{(1)}(z)=A_{0} e^{-i \omega t \pm i k z \pm i \kappa^{ \pm}\left(z-z_{ \pm}\right)} .
$$


Expanding to linear order in $\kappa^{ \pm}$we obtain

$$
A_{ \pm}^{(1)}(z)=A_{ \pm}^{(0)}(z)\left[1 \pm i \kappa^{ \pm}\left(z-z_{ \pm}\right)\right] .
$$

The above expression evaluated at $z=z_{ \pm}$simplifies to

$$
A_{ \pm}^{(1)}\left(z_{\mp}\right)=A_{ \pm}^{(0)}\left(z_{\mp}\right)\left(1 \pm i \kappa^{ \pm} L\right) .
$$

Further comparison of Equations (2.38) and (2.34) (with $z=z_{\mp}$ ) allows one to identify two complex loss/gain coefficients, $\kappa^{ \pm}$, as follows

$$
\kappa^{+}=\frac{1}{2 L i} \int_{-\infty}^{\infty} d p_{z} F\left(p_{z}\right) \int_{0}^{L} d z^{\prime} e^{-i k z^{\prime}} \sin g z^{\prime} \int_{0}^{z^{\prime}} d z^{\prime \prime} \sin g z^{\prime \prime} e^{i\left(z^{\prime}-z^{\prime \prime}\right) m \gamma \omega / p_{z}+i k z^{\prime \prime}}
$$

and

$$
\kappa^{-}=\frac{1}{2 L i} \int_{-\infty}^{\infty} d p_{z} F\left(p_{z}\right) \int_{0}^{L} d z^{\prime} e^{-i k z^{\prime}} \sin g z^{\prime} \int_{z^{\prime}}^{L} d z^{\prime \prime} \sin g z^{\prime \prime} e^{i\left(z^{\prime}-z^{\prime \prime}\right) m \gamma \omega / p_{z}+i k z^{\prime \prime}}
$$

Apart from a simple integration over $z^{\prime}$ and $z^{\prime \prime}$, we have found the complex coefficient, $\kappa^{ \pm}$, for waves propagating parallel and antiparallel to the particle beam. The imaginary part of $\kappa^{ \pm}$describes either spontaneous amplification or degradation (depending on its sign) of the optical mode. In the next section, the above integrations will be carried out explicitly and the linear gain/loss coefficients will be calculated in a closed form.

\section{LINEAR LOSS/GAIN COEFFICIENT - ACCELERATION RATE}

On carrying out the two spatial integrations in Equations (2.39) and (2.40), the expressions for the complex gain, $\kappa^{ \pm}$, reduce to

$$
\begin{aligned}
\kappa^{+}= & \frac{L}{16} \int_{-\infty}^{\infty} d p_{z} F\left(p_{z}\right)\left\{\frac{1}{\mu^{-}}\left[N\left(\mu^{+}\right)-N\left(\mu^{-}\right)-N(2 g)+N(0)\right]\right. \\
& +\frac{1}{\mu^{+}}\left[N\left(\mu^{-}\right)-N\left(\mu^{+}\right)+N(0)-N(-2 g)\right] \\
& -e^{-2 i k L} \frac{1}{\mu^{-}}\left[N\left(\nu^{-}\right)-N\left(\nu^{+}\right)+N(2 k+2 g)-N(-2 k)\right] \\
& \left.-e^{-2 i k L} \frac{1}{\mu^{+}}\left[N\left(\nu^{-}\right)-N\left(\nu^{+}\right)+N(2 k)-N(2 k-2 g)\right]\right\}
\end{aligned}
$$


and

$$
\begin{aligned}
\kappa^{-}= & \frac{L}{16} \int_{-\infty}^{\infty} d p_{z} F\left(p_{z}\right)\left\{\frac{1}{\nu^{-}}[N(2 g)-N(0)-N(2 g-2 k)+N(-2 k)]\right. \\
& -\frac{1}{\nu^{+}}[N(0)-N(-2 g)-N(-2 k)+N(-2 k-2 g)] \\
& -e^{-i \nu^{-} L} \frac{1}{\nu^{-}}\left[N\left(\nu^{+}\right)-N\left(\nu^{-}\right)-N\left(\mu^{+}\right)+N\left(\mu^{-}\right)\right] \\
& \left.-e^{-i \nu^{+} L} \frac{1}{\nu^{+}}\left[N\left(\nu^{+}\right)-N\left(\nu^{-}\right)-N\left(\mu^{+}\right)+N\left(\mu^{-}\right)\right]\right\}
\end{aligned}
$$

where

$$
\mu^{ \pm}=m \gamma \omega / p_{z}-k \pm g, \nu^{ \pm}=m \gamma \omega / p_{z}+k \pm g
$$

and

$$
N(x)=\frac{e^{i x L / 2}}{L / 2} \frac{\sin (x L / 2)}{x L / 2} .
$$

Using our definition of $\kappa^{ \pm}$the linear gain coefficient is given by $\alpha^{ \pm}=-\operatorname{Im} \kappa^{ \pm}$, and we observe that the function

$$
\Gamma(x) \equiv \operatorname{Im} \frac{N(x)}{x}=\left(\frac{\sin (x L / 2)}{x L / 2}\right)^{2}
$$

is the characteristic form occurring in diffraction theory, with the principal maximum at $x=0$. The remaining terms in curly brackets in Equations (3.1) and (3.2) do not have this feature and their contribution can be neglected relative to the terms containing $\Gamma$. Therefore, Equations (3.1) and (3.2) simplify to

$$
\alpha^{+}=\frac{L}{16} \int_{-\infty}^{\infty} d p_{z} F\left(p_{z}\right)\left[\Gamma\left(\mu^{+}\right)+\Gamma\left(\mu^{-}\right)\right]
$$

and

$$
\alpha^{-}=-\frac{L}{16} \int_{-\infty}^{\infty} d p_{z} F\left(p_{z}\right)\left[\Gamma\left(\nu^{+}\right)+\Gamma\left(\nu^{-}\right)\right]
$$

Since the quantities $g, k$ and $p_{z}$ are positive, for the chosen geometry, only $\mu^{-}$and $\nu^{-}$ pass through zero. Therefore, the remaining terms, $\Gamma\left(\mu^{+}\right)$and $\Gamma\left(\nu^{+}\right)$can be neglected when evaluated far from the maximum compared to the $\Gamma(x)=1,(x=0)$ term. Using the above argument, Equations (3.6) and (3.7) become

$$
\alpha^{+}=\frac{\pi}{4} n \int_{-\infty}^{\infty} d p_{z} Q^{2} \frac{p_{z}}{m \gamma} \frac{\partial \Lambda}{\partial p_{z}} L \Gamma\left(\mu^{-}\right)
$$


and

$$
\alpha^{-}=-\frac{\pi}{4} n \int_{-\infty}^{\infty} d p_{z} Q^{2} \frac{p_{z}}{m \gamma} \frac{\partial \Lambda}{\partial p_{z}} L \Gamma\left(\nu^{-}\right)
$$

where the explicit form of the kernel $F$ is used.

Imposing a resonant condition $\nu^{-}=0$ in Equation (3.9) fixes the wavevector of the optical mode as follows:

$$
g=m \gamma k c / p_{z}+k
$$

where

$$
p_{z}=m \gamma \beta c \text {. }
$$

Assuming the extreme relativistic limit, $\beta \rightarrow 1$, for the proton beam yields the following resonance condition:

$$
\lambda=2 \ell .
$$

One can summarize the above condition with the following statement: pumping the beam with the electromagnetic wave of twice the superlattice period would result in the maximum rate of energy extraction from the wave, given here by $\alpha^{-}$, which is the essence of the accelerating scheme presented.

One can notice in passing that $\alpha^{+}$corresponds to the lasing process, when the forward propagating wave is being amplified by the energy drawn from the beam (the FEL effect). The corresponding resonant condition is given by $\mu^{-}=0$, which yields a well-known scaling relation between the undulator period and the amplified wavelength:

$$
\lambda=\frac{\ell}{2 \gamma^{2}} .
$$

From now on, we are interested only in the inverse FEL situation; therefore we will simplify further notation by $\alpha \equiv \alpha^{-}$and $\nu \equiv \nu^{-}$.

One can notice that, apart from a slowly varying function $F$, the remaining functions occurring in the integrand in Equation (3.9), namely, $\Lambda$ and $\Gamma$ are sharply peaked functions of momentum characterized by the respective widths $\left(\frac{\Delta p}{p}\right)_{\Lambda}$ and $\left(\frac{\Delta p}{p}\right)_{\Gamma}$. Here the width of the initial momentum distribution, $\Lambda$, was introduced in a standard way: ${ }^{8}$

$$
\left(\frac{\Delta p}{p}\right)_{\Lambda}=\left[\left(p_{z}^{2} \frac{\partial \Lambda}{\partial p_{z}}\right)_{\max }\right]^{-1 / 2}
$$

Similarly, the width of $\Gamma$ is governed by the following simple ratio:

$$
\left(\frac{\Delta p}{p}\right)_{\Gamma}=\frac{\ell}{L}
$$


Now one can compare relative sharpness of both functions; $\Lambda$ and $\Gamma$. The relative momentum spread is typically of order $10^{-4}$. Assuming a superlattice modulation period of $500 \AA$ and crystal length of $5 \mathrm{~cm}$ allows one to evaluate the width of $\Gamma$. Both characteristic widths can be summarized as follows:

$$
\left(\frac{\Delta p}{p}\right)_{\Lambda}=10^{-4}, \quad\left(\frac{\Delta p}{p}\right)_{\Gamma}=10^{-6} .
$$

The integration in Equation (3.9) is carried out assuming that the sharper function, namely $\Gamma$, is approximated by the $\delta$-function according to the following asymptotic relationship:

$$
\lim _{L \gg \ell} \frac{L}{2 \pi} \Gamma(\nu)=\delta(\nu) .
$$

Applying the resonant condition, described by Equations (3.10)-(3.1), and assuming $\beta \rightarrow 1$ reduces the gain/loss coefficient to the following simple expression:

$$
\alpha=-\frac{\pi}{2} n \frac{Q^{2}}{m} \gamma \ell\left(\frac{\Delta p}{p}\right)_{\Lambda}^{-2} .
$$

This above final result will serve as a starting point for further feasibility discussion.

\section{THREE-WAVE MIXING — PHYSICAL PICTURE}

According to the model calculation presented, spontaneous bunching of the proton beam as it channels through a superlattice and interacts with the electromagnetic wave results in energy flow from the wave to the beam. This particular kind of particle density fluctuation, $h$, has the form of a propagating plane wave of the same frequency, $\omega$, as the emitted electromagnetic wave. The phase velocity of the moving bunch matches the velocity of protons in the beam. Therefore, the quantity $\gamma m \omega / p_{z} \equiv k_{b}$ represents the wavevector of the propagating particle density bunch. Keeping in mind that the periodicity of the undulator represents a static wave with a wavevector $g$, and that $k$ is the wavevector of the electromagnetic wave, we can analyze our results in the language of three-wave mixing.

The resonant denominators appearing in the final expressions for the linear gain/loss coefficients, Equation (3.3), i.e., $\mu^{ \pm}=m \gamma \omega / p_{z}-k \pm g$ (forward-propagating wave) and $\nu^{ \pm}=m \gamma \omega / p_{z}+k \pm g$ (backward-propagating wave), can be identified with the frequency - wavevector conservation conditions for a three wave-mixing process. From this point of view, the FEL or the inverse FEL reduce to an Umklapp process ${ }^{8}$ involving (1) the propagating "bunch" in the proton density, (2) the pumping electromagnetic wave, and (3) the static periodic field of the undulator. Matching of frequencies for the two "dynamic modes" assures "energy" conservation. Furthermore "momentum" conservation of all three modes (static and dynamic) yields the $\mu^{-}=0$ (FEL) 
and $\nu^{-}=0$ (inverse FEL) conditions. The last condition is equivalent to a momentum "recoil", $g$, between the particle density "bunch" and the electromagnetic wave, i.e., an Umklapp process. One can see immediately that the resonant denominator, $\nu^{-}$, is responsible for the negative gain (deamplification of the backward propagating wave), where a four-momentum $(0, g)$ is transferred from the backward-propagating wave to the forward moving proton bunch.

\section{FEASIBILITY ASSESSMENT}

In this section we will discuss the feasibility of the proposed scheme by considering [110] planar channeling in a strain modulated $\mathrm{Si}$ crystal ${ }^{9}$. We write the undulator period as $\ell=N d$, where $d=1.92 \AA$ is the spacing between successive lattice planes and $N$ is the number of such planes. The strain modulation, of course, requires a second component, such as Ge; however, we will use the parameters of Si for convenience.

The magnetic field of the undulator would be equivalent to the electric field of the strain-modulated crystal lattice (i.e., it would impart the same transverse velocity to the channeling particles). The equivalence is given by:

$$
B=\phi_{1} x_{1} .
$$

According to Reference 9, the harmonic part of the crystal field potential for [110] planar channeling in $\mathrm{Si}$ is $e \phi_{1}=36 \mathrm{eV} \AA^{-2}$. Assuming a strain modulation amplitude of 0.1 A leads to an enormous magnetic field, $\mathbf{B}=1.2 \times 10^{6} \mathrm{G}$.

While channeling along the path, relativistic particles undergo transverse harmonic oscillations, an analog of betatron oscillations, because of the crystal field potential, with the characteristic frequency $\omega_{\beta}=\sqrt{e \phi_{1} / m}$. One can see from Equation (2.29) that if the angular velocity of a particle traversing the strain modulated path, $\omega=$ $2 \pi v_{\perp} / \ell$, approaches $\omega_{\beta} / \sqrt{\gamma}$ (Doppler shifted betatron frequency), the undulator parameter, $Q$, has a resonance $(U \rightarrow 1)$, which would enormously enhance the gain/loss coefficient. However, the excessive growth of the undulator parameter would soon result in a rapid dechanneling of the particles. One can see this easily if $Q$ is rewritten in the following form

$$
Q=\frac{e}{c} \frac{v_{\perp}}{v_{\|}}
$$

where $v_{\perp}, v_{\|}$are transverse and longitudinal components of the particle velocity, respectively.

The following simple physical criterion allows one to estimate the maximum value of $Q$. Dechanneling will occur if the transverse kinetic energy of the particle exceeds the binding energy of the harmonic potential (that is, a particle leaves the channel). If the maximum transverse velocity of a channeling particle is $v_{\perp}$ and $a$ is the distance 
between adjacent channels (for [110] channeling in Si where $a=5 \AA$ ), the above condition can be written as follows:

$$
v_{\perp}^{2} \geq \frac{e \phi_{1}}{m}\left(\frac{a}{2}\right)^{2} .
$$

Equation (5.2) and the equality sign in Equation (5.3), fix the maximum allowed value of the undulator parameter:

$$
Q^{\max }=\frac{e}{c} \frac{a}{2} \sqrt{\frac{e \phi_{1}}{m c^{2}}} \frac{\gamma}{\sqrt{\gamma^{2}-1}} .
$$

The above expression can be evaluated for relativistic protons channeling through our model superlattice as

$$
Q^{\max }=7.5 \frac{\gamma}{\sqrt{\gamma^{2}-1}} \times 10^{-24} \mathrm{~cm}^{1 / 2} \mathrm{~g}^{1 / 2}
$$

Now, one can evaluate Equation (3.18) assuming only one proton - by assigning $n$ to be an inverse area of the channeling plane per one particle for typical values of the beam concentration, $n=10^{16} \mathrm{~cm}^{-2}$. In this way, $\alpha$ describes the rate of optical amplitude depletion per one particle - the acceleration rate. Assuming a proton beam of initial energy $50 \mathrm{MeV}(\gamma=1.0533), \ell=500 \AA$, and $\left(\frac{\Delta p}{p}\right)_{\Lambda}=10^{-4}$, the calculation yields the following acceleration rate:

$$
\alpha=2.68 \times 10^{-3} \mathrm{~cm}^{-1} .
$$

The nominal acceleration efficiency in units of $\mathrm{MeV} / \mathrm{m}$ will, obviously, depend on the energy density of the actual optical cavity. Here the $\nu^{-}=0$ resonant condition translates into the following optical wavelength constraint:

$$
\lambda=\left(1+\frac{1}{\beta}\right) \ell,
$$

which in our numerical example fixes the accelerating optical wavelength at $\lambda=209$ $\mathrm{nm}$. The recent advances in high-power laser technology based upon diode-laserpumped solid-state lasers ${ }^{10}$ promise a power of a few MW, optically focused to provide energy densities of $\mathcal{E}^{\max }=10^{10} \mathrm{~V} / \mathrm{m}$, where $\mathcal{E}^{\max }$ is the electric field amplitude of the standing cavity mode. This combined with Equation (5.7), sets the final accelerating efficiency equivalent to an accelerating gradient of $2.68 \mathrm{GeV} / \mathrm{m}$.

\section{CONCLUSIONS}

The final acceleration rate evaluated numerically in Equation (5.7) seems to be quite substantial. Practical feasibility of the scheme rests on availability of a high-density 


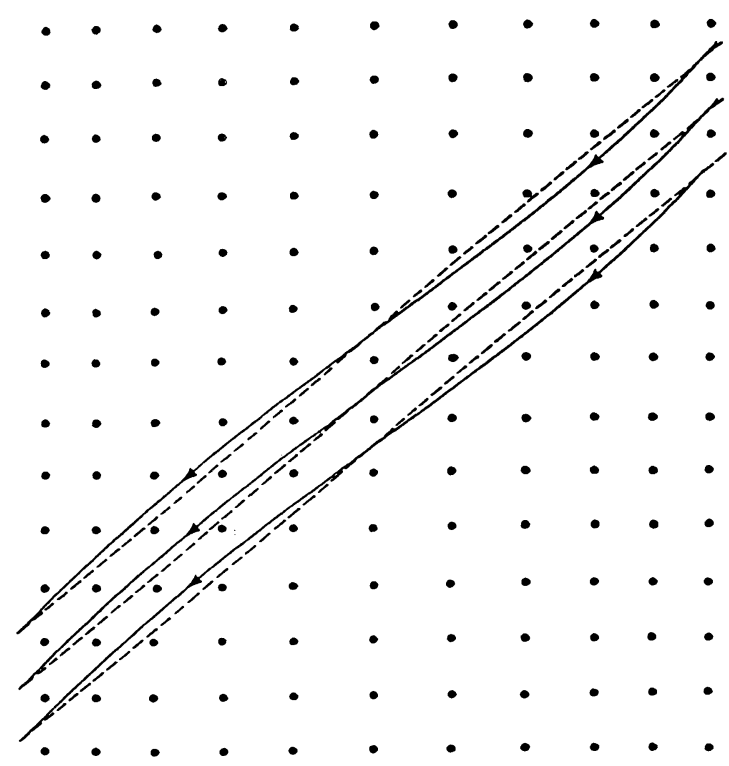

FIGURE 2: An infinite array of parallel channels in a strain-modulated superlattice. The phase shift in the crystal field potential between two neighboring channels, $\rho$, is given by $\rho=\frac{a \pi}{\ell}$.

optical mode pumped by a high-power laser. Furthermore, there is some radiation damage and thermal heating of the crystal associated with proton channeling. One could apply the beam to a rapidly spinning crysta ${ }^{11}$ so that the average power density could be reduced to alleviate this problem.

\section{ACKNOWLEDGEMENT}

The author wishes to thank Bob Noble for discussion and his helpful comments on transient vs. steady-state solution of crystal channeling kinematics.

\section{APPENDIX}

We will generalize the treatment given in Section 2, Equations (2.19)-(2.24), to a situation depicted schematically in Figure 2. Now there is a constant phase shift, $\rho$, between the crystal fields of two neighboring channels. In this case the kinetic equation, Equation (2.19), can be modified as follows

$$
\left(\frac{\partial}{\partial t}+v_{z} \frac{\partial}{\partial z}\right) h^{j}(z, t)=M \frac{\partial A}{\partial z} \sin (g z+j \rho) .
$$


Here $j$ is the index of the channel, which discretizes the variable $x$ as follows: $x=a j$. Furthermore we denote

$$
v_{z}=\frac{p_{z}}{m \gamma}
$$

and

$$
M=v_{z} Q \frac{\partial \Lambda}{\partial p_{z}} .
$$

Assuming an $x$-dependent A-field of the form

$$
A(x, z, t)=A(z) e^{-i \omega t+i k_{x} x},
$$

we seek a solution for $h$ in the following form

$$
h^{j}(z, t)=h^{j}(z) e^{-i \omega t} .
$$

Substituting the above expression in Equation (A.1), one can rewrite it as follows:

$$
\left(v_{z} \frac{\partial}{\partial z}-i \omega\right) h^{j}(z)=\frac{M}{2 i}\left(e^{i g z+i\left(k_{x} a+\rho\right) j}-e^{-i g z+i\left(k_{x} a-\rho\right) j}\right) \frac{\partial A}{\partial z} .
$$

Therefore the $j$-dependence of our solution can be written explicitly as

$$
h^{j}(z)=h_{+}(z) e^{i\left(k_{x} a+\rho\right) j}+h_{-}(z) e^{i\left(k_{x} a-\rho\right) j},
$$

where the functions $h_{+}$and $h_{-}$are governed by the following pair of equations, equivalent to Equation (A.2):

$$
\left(v_{z} \frac{\partial}{\partial z}-i \omega\right) h_{+}(z)=\frac{M}{2 i} e^{i g z} \frac{\partial A}{\partial z}
$$

and

$$
\left(v_{z} \frac{\partial}{\partial z}-i \omega\right) h_{-}(z)=-\frac{M}{2 i} e^{-i g z} \frac{\partial A}{\partial z} .
$$

The net transverse current in the crystal, induced by $h_{+}$and $h_{-}$, couples to the A-field through the following wave equation

$$
\left(\frac{\partial^{2}}{\partial x^{2}}+\frac{\partial^{2}}{\partial z^{2}}+k^{2}\right) A(x, z)=4 \pi n_{0} \int_{-\infty}^{\infty} d p_{z} v_{z} Q \sum_{j} \delta(x-j a) \sin (g z-j p) h^{j}(z)
$$


where

$$
k^{2}=k_{x}^{2}+k_{z}^{2}=(\omega / c)^{2} .
$$

Substituting according to Equation (A.3) and representing the $\delta$-function as an integral form, $\delta(x)=\int_{-\infty}^{\infty} d k_{x} e^{i k_{x} x}$, one can rewrite Equation (A.5) as follows:

$$
\begin{gathered}
\left(\frac{\partial^{2}}{\partial z^{2}}+k_{z}^{2}\right) A(z)=4 \pi n_{0} \int_{-\infty}^{\infty} d p_{z} v_{z} Q \frac{1}{2 i a} \\
\times\left(h_{-} e^{-i g z}-h_{+} e^{i g z}-h_{-} e^{-i g z-i(2 \rho / a) x}-h_{+} e^{i g z+i(2 \rho / a) x}\right) .
\end{gathered}
$$

Obviously for a channeling particle $x \ll a$ and therefore $e^{i(2 \rho / a) x} \cong 1$; Equation (A.7) then becomes

$$
\left(\frac{\partial^{2}}{\partial z^{2}}+k_{z}^{2}\right) A(z)=4 \pi n_{0} \int_{-\infty}^{\infty} d p_{z} v_{z} Q \frac{1}{a}\left(h_{-}+h_{+}\right) \sin g z
$$

Similarly from Equation (A.8) one obtains

$$
\left(v_{z} \frac{\partial}{\partial z}-i \omega\right)\left[h_{+}(z)+h_{-}(z)\right]=M \frac{\partial A}{\partial z} \sin g z
$$

One can see immediately that redefining; $h_{+}+h_{-}=h$, in Equations (A.7) and (A.8) reduces them to the initial pair of equations used previously, namely, Equations (2.19) and (2.22). Therefore, our case $(x \ll a)$ is equivalent to the one treated in Section 2, and the same final expression for the linear gain/loss holds even in the case of a finite phase shift in the crystal potential between neighboring channels.

\section{REFERENCES}

1. R. B. Palmer, Part. Accel. 11, 81 (1980).

2. J. A. Ellison, S. T. Picraux, W. R. Allen and W. K. Chu, Phys. Rev. B 37, 7290 (1988).

3. S. Krinsky and J. M. Wang, Part. Accel., 17, 109 (1985).

4. B. L. Berman, S. Datz, R. W. Fearick, R. L. Swent, R. H. Pantell, H. Park, J. O. Kephart and R. K. Klein, Nucl. Instrum. Meth. B 2, 90 (1984).

5. J. A. Ellison, Phys. Rev. B 18, 5948 (1978).

6. M. A. Kumakhov, Sov. Phys. JEPT 4, 781 (1977).

7. L. D. Landau and E. M. Lifshitz, Electrodynamics of Continuous Media (Pergamon Press, Oxford, 1960), Ch. XV.

8. E. M. Lifschitz and L. P. Pitaevskii, Physical Kinetics (Pergamon Press, Oxford, 1981), Ch. VI.

9. S. Datz, R. W. Fearick, H. Park, R. H. Pantell, R. L. Swent, J. O. Kephart and B. L. Berman, Nucl. Instrum. Meth. B 2, 74 (1984).

10. S. Basu and R. L. Byer, Optic Letters 13, 458 (1988).

11. R. H. Pantell and M. J. Alguard, J. Appl. Phys. 50, 798 (1979). 